\title{
Carvacrol and thymol as potential preservatives against Aspergillus in maize grains
}

\author{
Jade Cardôso Lima1, Sávio Marcelino Gomes', Edeltrudes de Oliveira Lima ${ }^{3}$, Fillipe de Oliveira Pereira², \\ Igara Oliveira Lima ${ }^{1}$
}

${ }^{1}$ Microbiology Laboratory, Academic Health Unit, Health and Education Center Federal University of Campina Grande, Cuité, Brazil, ${ }^{2}$ Biochemistry Laboratory, Academic Health Unit, Education and Health Center, Federal University of Campina Grande, Cuité, Brazil, ${ }^{3}$ Mycology Laboratory, Department of Pharmaceutical Science, Health Sciences Center, Federal University of Paraıba, João Pessoa, Brazil

\section{A B S TR A C T}

This study investigated the antifungal potential of carvacrol and thymol against strains of Aspergillus flavus, A. fumigatus, and A. niger. Minimum inhibitory concentration (MIC) and minimum fungicidal concentration (MFC) were determined. The constituents' effect was also evaluated for possible preservative activity against fungal mycelial growth in a maize grain contamination model. At the concentrations tested, carvacrol inhibited the growth of all strains used; thymol did not inhibit the growth of the $A$. flavus strain tested. For both phytochemicals, the MFCs determined for the A. niger strain were equivalent to the value of their MICs. However for $A$. flavus and A. fumigatus, the MFC determined for carvacrol was four times its MIC value. At the concentrations tested, these phytochemicals were able to slow fungal mycelial growth, though carvacrol proved to be more effective than thymol in the maize grain contamination model.

Keywords: Aspergillus; Maize; Monoterpenes; Foodo; Phytoconstituents

\section{INTRODUCTION}

Fungi, being extensively abundant around the planet, are extremely important for the food, beverage and pharmaceutical industries where they play both ecological and economic roles (Kavanagh, 2017). Of the filamentous fungi, the genus Aspergillus is principally responsible for food contamination, such as in cereals where they cause deterioration, reduction in nutritional values, alteration of sensory quality, and since certain species produce mycotoxins they have become a matter of public health (Zulkifli and Zakaria, 2017).

Maize, also known as corn, is one of the principal cereals produced in the world (Zea mays L.), and is used in both animal and human feeding. Brazil occupies a prominent place in world maize production (Ranum, Peña-Rosas, and Garcia-Casal, 2014). However, maize production suffers large losses annually from several causes, one of these is microorganisms such as fungi of the genus Aspergillus spp. (Munkvold et al., 2018).
Food-borne diseases are a growing problem in public health, and to achieve successful pathogen control in the manufacture and storage of food products requires the use of preservation techniques (Hyldgaard et al., 2012). Thus, natural products have been increasingly studied and used in alternative products to control microorganisms (Oliveira et al., 2014). Terpenes are considered the most common class of substances found in plants. When isolated from essential oils, the phytochemicals carvacrol and thymol are excellent examples of phenolic monoterpene isomers which present antimicrobial activity, (Abbaszadeh et al., 2014).

This study aimed to investigate the in vitro antifungal activity of carvacrol and thymol against certain strains of Aspergillus, and to test their applicability as protective food ingredients in a maize grain contamination model.

\section{MATERIAL AND METHODS}

Three Aspergillus spp. fungal strains (A. flavus ATCC-13013; A. fumigatus ATCC-40640; and A. niger LM-27) were

\footnotetext{
*Corresponding author:

Fillipe de Oliveira Pereira, Biochemistry Laboratory, Academic Health Unit, Education and Health Center, Federal University of Campina Grande, Cuité, Brazil. E-mail: fillipefop@yahoo.com.br
} 
provided by the Mycology Laboratory of the Department of Pharmaceutical Sciences, at the Federal University of Paraiba. The fungal strains were incubated in Sabouraud Dextrose Agar (SDA- Difco ${ }^{\circledR}$ ) for 7 days, at $28^{\circ} \mathrm{C}$. The fungal colonies were suspended in a sterile saline $(0.9 \%$ $\mathrm{NaCl}$ ) solution. The suspension density of each strain was adjusted to a value of $70 \% \pm 2$ transmittance, which corresponds to an inoculum of approximately $1-5 \times 10^{6}$ colony-forming units per milliliter $(\mathrm{CFU} / \mathrm{mL})$, using a spectrophotometer at $530 \mathrm{~nm}$ (CLSI, 2002).

MIC determination was performed by microdilution technique using RPMI medium (CLSI, 2002). The drug emulsions were prepared dissolving previously calculated amounts of the phytochemicals carvacrol and thymol (Sigma Aldrich ${ }^{\circledR}$, Brazil) in $50 \mu$ l of Tween 80 (Polysorbate 80 ), and RPMI medium. The drugs were tested from $16 \mu \mathrm{g} / \mathrm{mL}$ to $2048 \mu \mathrm{g} / \mathrm{mL}$. Three controls were performed: a fungal control to observe the viability of the strain, containing only the RPMI medium and inoculum; a sterility control, using only the culture medium without inoculum; and a control to verify possible interference in the results containing the culture medium, inoculum, and Tween 80 at the same concentration used for drug solubilization. The plates were sealed and incubated at $28^{\circ} \mathrm{C}$ for up to 7 days for reading. The lowest concentration of these drugs capable of inhibiting fungal growth by $100 \%$ as observed in the wells was determined as the MIC value.

To determine the MFCs, an aliquot of $10 \mu \mathrm{L}$ was removed from each well presenting no fungal growth and then seeded on a plate containing SDA and incubated at $28^{\circ} \mathrm{C}$ for 7 days. The CFU value was then determined and the MFC was considered as the lowest seeded concentration presenting less than 3 CFUs (Klepser and Pfaller, 1998; Espinel-Ingroff et al., 2002).

Analysis of test drug interference on fungal mycelial growth was performed by measuring radial mycelial growth in SDA medium and using MIC and 2xMIC as the test drug concentrations. A negative control (absent drug) was also performed. The entire system was incubated at $28^{\circ} \mathrm{C}$ for a total of 7 days; radial mycelial growth was recorded daily (Khan and Ahmad, 2011).

The maize (corn), fit for human consumption, was provided by the Department of Agriculture of CuitéPB city. The grains were dried at $40^{\circ} \mathrm{C}$ for 48 hours to reach approximately $14 \%$ moisture. Only grains that did not appear to be diseased were used. The healthy maize grains were packed into Erlenmeyers and autoclaved for 15 minutes at $121^{\circ} \mathrm{C}$ (Dambolena et al., 2010). The drugs were distributed to test tubes at the test concentrations (MIC and 2xMIC), diluted in sterile distilled water, the autoclaved grains (immersed for 1 minute in $200 \mu \mathrm{L}$ of the fungal inoculum at $10^{6} \mathrm{CFUs} / \mathrm{mL}$ ) were then transferred to the tubes, being 10 grains of possibly infected maize in each tube. The tubes were then incubated at $28^{\circ} \mathrm{C}$ for 7 days and visual analysis was performed. The next step was to distribute the grains from the tubes to Petri dishes containing potato dextrose agar (Difco ${ }^{\circledR}$, Brazil) medium with chloramphenicol to determine the presence or absence of fungal growth; daily reading was performed. A fungal control containing only medium and infected maize grains, and a sterile control using only medium and sterile grains were also performed. Another control containing medium, infected maize grains, and Tween 80 (at the same concentration used for drug solubilization to verify its noninterference in the results) was also performed.

The effect of drug vapors on infected grains was also evaluated. After an identical infection induction process, 10 grains were placed in disposable Petri dishes, each containing $90 \mathrm{~mm}$ filter paper soaked in the MIC and 2xMIC concentrations of the test drugs (inside the Petri dish lid). The plates were quickly sealed and incubated at $28^{\circ} \mathrm{C}$ for 24 hours. To identify the presence of infection (by growth in medium) the grains were transferred to Petri dishes with potato dextrose agar containing chloramphenicol (10 grains per plate), and then incubated at $28^{\circ} \mathrm{C}$ for 7 days; reading was done daily (Mennitti et al., 2010).

The experiments were performed in triplicate. MIC and MFC values were expressed as modal values, and the mycelial growth results were compared statistically using the unpaired t-test. The results of the maize grain conservation assay were expressed as mean $\pm \mathrm{SD}$ of percentage of infected grains; the data were compared statistically using the Fischer test. A p $<0.05$ was considered significant.

\section{RESULTS AND DISCUSSION}

The MICs for carvacrol found in this study for the strains of $A$. flavus, $A$. fumigatus and $A$. niger were respectively $512 \mu \mathrm{g} / \mathrm{mL}, 256 \mu \mathrm{g} / \mathrm{mL}$, and $64 \mu \mathrm{g} / \mathrm{mL}$. For thymol, no MIC at the any concentration tested for the $A$. flavus strain was observed. For the $A$. fumigatus strain the MIC was $1024 \mu \mathrm{g} / \mathrm{mL}$ and for $A$. niger it was $128 \mu \mathrm{g} / \mathrm{mL}$. The fungal control containing only RPMI medium and inoculum presented growth in all wells of the microdilution plate; as did the control containing RPMI, inoculum, and Tween 80 medium (demonstrating the absence of Tween 80 interference in the experiment. There was no growth in the sterility control which contained only the culture medium, guaranteeing a viable execution of the method. Once the MICs were determined, the next step was to determine the minimum fungicidal concentrations (MFCs) 
for strains that had MICs lower than $1000 \mu \mathrm{g} / \mathrm{mL}$. The MFC determined for the $A$. niger strain was equivalent to its MIC value, whereas for the $A$. flavus and $A$. fumigatus strains the MFC values were four times the MIC values.

The effect on fungal mycelial growth of the two phytoconstituents was also evaluated. In Figs. 1 and 2 it can be seen that carvacrol (MIC and 2xMIC) was able to reduce radial mycelial growth for the $A$. fumigatus and A. flavus strains tested ( $\mathrm{p}<0.05)$. As in the MFC testing, thymol was only applied against the $A$. niger strain, and it may be observed in Fig. 3, both phytochemicals presented similar values at the concentrations tested, also presenting a standard deviation for growth on the fifth day of reading $(\mathrm{p}<0.05)$.

Monoterpene antifungal activity has been observed in other studies; demonstrating that the antimicrobial action mechanism of phenolic compounds such as carvacrol and thymol are similar; interaction with cell membranes causing structural and functional damage (Perricone et al., 2015). In a study by Zabka et al. (2013), evaluating the efficacy of 21 phenolic compounds against strains of filamentous and toxigenic (A. flavus and A. fumigatus), the MIC values for thymol varied between $76-255 \mu \mathrm{g} / \mathrm{mL}$. For carvacrol, the MIC values varied between 131-262 $\mu \mathrm{g} / \mathrm{mL}$. Both were considered promising phenols, since of the compounds tested they presented the greatest efficacy.

Since maize (when infected by fungi) constitutes an important source of inoculum whose pathogens may cause seed rot, the next step was to study potential preservative activity for thymol and carvacrol in a maize contamination model (Fig. 4). The grains of maize were stored in solutions containing the test drugs at the desired concentrations, due to the MIC values already found (Fig. 5) thymol was only tested on the $A$. niger strain. After 7 days in storage, grains not appearing to be infected were transferred to Petri dishes containing potato dextrose agar, the following graphs demonstrate the fungal growth in the grains as a function of time.

During storage the control tubes presented visible fungal growth, whereas the tubes containing the carvacrol solution and grains (possibly infected with A. flavus and $A$. fumigatus), presented no growth. However when these grains were transferred to Petri dishes, visible fungal growth was observed at two days of incubation, indicating that carvacrol presented inhibitory activity only while in contact with the grains. For the $A$. niger strain, in the maize grain contamination model neither carvacrol nor thymol presented inhibitory activity in the concentrations studied.

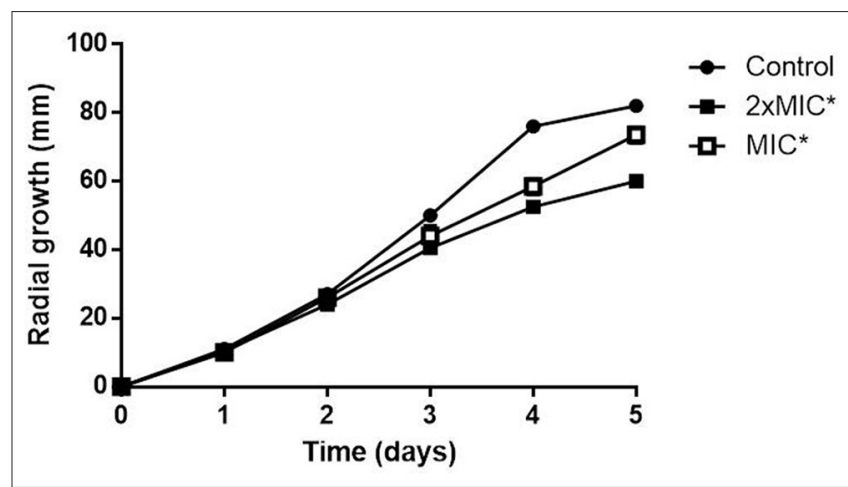

Fig 1. Radial mycelial growth (mm) of Aspergillus fumigatus in the presence and absence of carvacrol. *Compared with control on fifth day $(p<0.05)$.

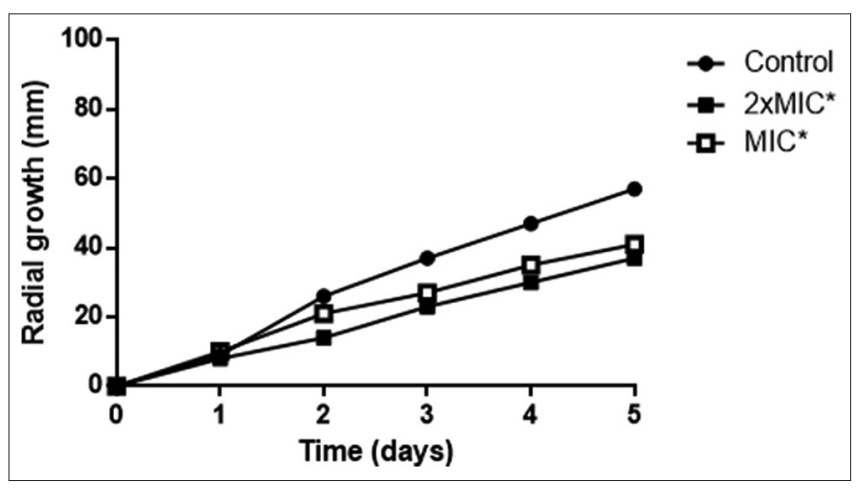

Fig 2. Radial mycelial growth ( $\mathrm{mm}$ ) of Aspergillus flavus in the presence and absence of carvacrol. ${ }^{*}$ Compared with control on fifth day $(p<0.05)$.

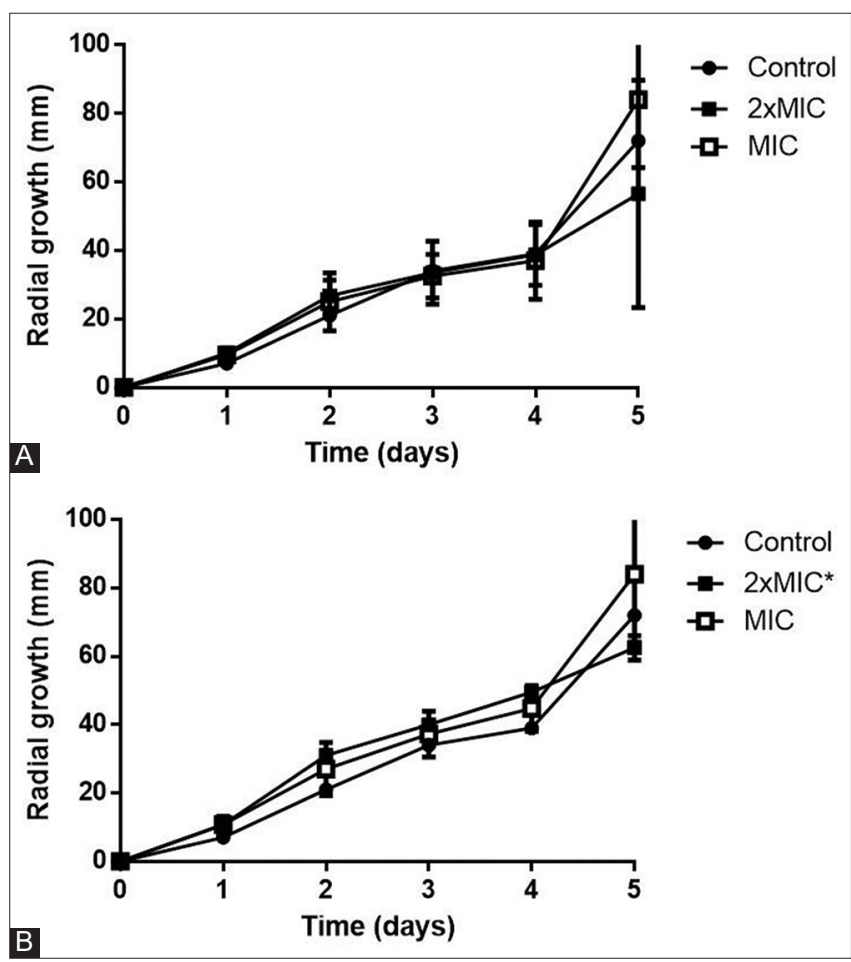

Fig 3. Radial mycelial growth ( $\mathrm{mm}$ ) of Aspergillus niger in the presence and absence of carvacrol (A) and thymol (B). ${ }^{*}$ Compared with control on fifth day $(p<0.05)$. 


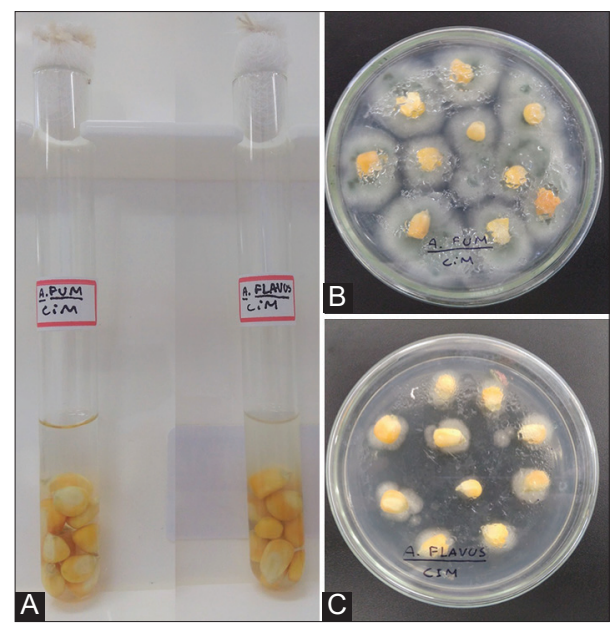

Fig 4. Maize grains infection test. A: Grains of maize in test tubes after 7 days of contact with sub-inhibitory test drug concentrations (no apparent infection). B and C: fungal growth in dextrose potato agar after 48 hours of incubation.

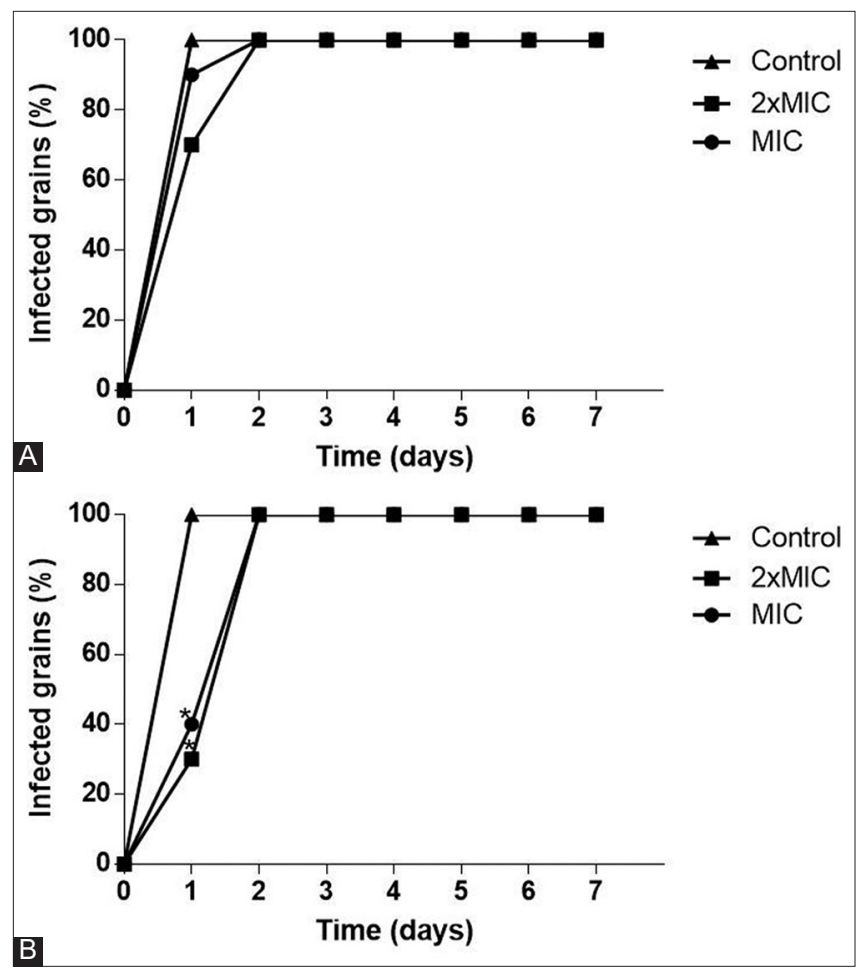

Fig 5. Effects of carvacrol concentrations on the growth of Aspergillus fumigatus (A) and Aspergillus flavus (B) on experimentally infected maize grains. ${ }^{*} p<0.05$ when compared to control (Fischer test), per day of analysis.

All of the tests involving with terpene vapors at both the MIC and 2xMIC concentrations presented fungal growth on the maize grains at two days of incubation; indicating the inefficiency of these terpene vapors as applied to the strains studied. Our results present relevant data and confirm the potential of carvacrol and thymol as food preservatives against Aspergillus spp. In a model of maize grains experimentally contaminated with $A$. flavus, Esper et al. (2014) tested Origanum vulgare essential oil and confirmed it as a good alternative for control and protection against Aflatoxin $\mathrm{B}_{1}$ in maize grains. The presence of mycotoxins in the present study was not evaluated, but this is a pertinent line of study that may be pursued in further work.

Despite the efficacy observed for essential oils and their constituents against food-borne pathogens in in vitro tests, the same efficacy in food products generally requires higher concentrations (Avila-Rosa et al., 2012). In this context, the use of drug vapors may be beneficial in food applications since (with vapor) there are fewer interactions affecting the sensory properties of the food. Thus, our results for terpene vapors and maize grain reveal that though the substances tested are quite volatile, they may suffer interference from various environmental and storage factors (Passone et al., 2013).

Consumers are increasingly aware of the effects that synthetic food additives can cause, and increasingly reject products with such additives. This creates demand for more natural products. Studies on the use of natural volatile compounds as alternatives are increasing. The choice of plants historically used in alternative medicine whose safety has been proven builds consumer confidence. Thus, purchases of "green products" bring the larger economic benefit of increasing demand for a wider range of plants (Tongnuanchan and Benjakul, 2014).

\section{CONCLUSIONS}

From the results we conclude that carvacrol presents in vitro antifungal activity against all of the strains of Aspergillus spp. tested under the evaluated conditions. Yet, at the concentrations used, thymol did not present activity for the A. flavus strain. In the maize grain contamination model, carvacrol proved to be more effective than thymol, which makes carvacrol a good choice for future studies concerning food applications. It is important to emphasize that more studies are needed in this area to understand interactions between monoterpenes and food matrices, synergistic interactions between essential oil components, and to reduce negative physicochemical and sensory effects on food characteristics.

\section{Author contributions}

Jade Cardôso Lima and Sávio Marcelino Gomes: developed the lab research and assisted in writing the article.

Edeltrudes de Oliveira Lima: provided the fungi used in this study, as well as technical advice.

Fillipe de Oliveira Pereira and Igara Oliveira Lima supervised this work in the following aspects: coordinating 
the biochemistry and microbiology laboratories, writing of the article, and interpretation of the data.

\section{REFERENCES}

Abbaszadeh, S., A. Sharifzadeh, H. Shokri, A. R. Khosravi and A. Abbaszadeh. 2014. Antifungal efficacy of thymol, carvacrol, eugenol and menthol as alternative agents to control the growth of food-relevant fungi. J. Mycol. Med. 2: 51-56.

CLSI. 2002. Reference Method for Broth Dilution Antifungal Susceptibility Testing of Filamentous Fungi (Approved Standard, No. M38 A. Vol. 22. Clinical and Laboratory Standards Institute, Europe.

Dambolena, J. S., M. P. Zunino, A. G. López, H. R. Rubinstein, J. A. Zygadlo, J. W. Mwangi, G. N. Thoithi, I. O. Kibwage, J. M. Mwalukumbi and S. T. Kariuki. 2010. Essential oils composition of Ocimum basilicum L. and Ocimum gratissimum L. from Kenya and their inhibitory effects on growth and fumonisin production by Fusarium verticillioides. Innov. Food Sci. Emerg. Technol. 11: 410-414.

Esper, R. H., E. Gonçalez, M. O. Marques, R. C. Felicio and J. D. Felicio. 2014. Potential of essential oils for protection of grains contaminated by aflatoxin produced by Aspergillus flavus. Front. Microbiol. 5: 269.

Espinel-Ingroff, A., A. Fothergill, J. Peter, M. G. Rinaldi and T. J. Walsh. 2002. Testing conditions for determination of, minimum fungicidal concentrations of new and established antifungal agents for Aspergillus spp.: NCCLS collaborative study. J Clin Microbiol. 40: 3204-3208.

Hyldgaard, M., T. Mygind and R. L. Meyer. 2012. Essential oils in food preservation: Mode of action, synergies, and interactions with food matrix components. Front. Microbiol. 3: 12.

Kavanagh, K. 2017. Fungi: Biology and Applications. $3^{\text {th }}$ ed. John Wiley and Sons, Chichester.

Khan, M. S. and I. Ahmad. 2011. In vitro antifungal, anti-elastase and anti-keratinase activity of essential oils of Cinnamomum, Syzygium and Cymbopogon species against Aspergillus fumigates and Trichophyton rubrum. Phytomedicine. 19: 48-55.

Klepser, M. E., E. J. Wolfe and M. A. Pfaller. 1998. Antifungal pharmacodynamic characteristics of fluconazole and amphotericin B against Cryptococcus neoformans. J. Antimicrob. Chemother. 41: 397-401.

Menniti, A. M., R. Gregori and F. Neri. 2010. Activity of natural compounds on Fusarium verticillioides and fumonisin production in stored maize kernels. Int. J. Food Microbiol. 136: 304-309.

Munkvold, G. P., L. Weieneth, R. Proctor, M. Busman, M. Blandino, A. Susca, A. Logrieco and A. Moretti. 2018. Pathogenicity of fumonisin-producing and nonproducing strains of Aspergillus species in section Nigri to maize ears and seedlings. Plant Dis. 102: 282-291.

Oliveira, J. L., E. V. R. Campos, M. Bakshi, P. C. Abhilash and L. F. Fraceto. 2014. Application of nanotechnology for the encapsulation of botanical insecticides for sustainable agriculture: prospects and promises. Biotechnol. Adv. 32: 1550-1561.

Passone, M. A., N. S. Girardi and M. Etcheverry. 2013. Antifungal and antiaflatoxigenic activity by vapor contact of three essential oils, and effects of environmental factors on their efficacy. LWT Food Sci. Technol. 53: 434-444.

Perricone, M., E. Arace, M. R. Corbo, M. Sinigaglia and A. Bevilacqua. 2015. Bioactivity of essential oils: A review on their interaction with food components. Front Microbiol. 6: 76.

Ranum, P., J. P. Peňa-Rosas and M. N. Garcia-Casal. 2014. Global maize production, utilization, and consumption. Ann. N. Y. Acad Sci. 1312: 105-112.

Tongnuanchan, P. and S. Benjakul. 2014. Essential oils: Extraction, bioactivities, and their uses for food preservation. J. Food Sci. 79: $1231-1249$.

Zabka, M. and R. Pavela. 2013. Antifungal efficacy of some natural phenolic compounds against significant pathogenic and toxinogenic filamentous fungi. Chemosphere. 93: 1051-1056.

Zulkifli, N. A. and L. Zakaria. 2017. Morphological and molecular diversity of Aspergillus from corn grain used as livestock feed. HAYATI J. Biosci. 24: 26-34. 